\title{
Coordinate actions of BMPs, Wnts, Shh and Noggin mediate patterning of the
} \section{dorsal somite}

\author{
Christophe Marcelle ${ }^{1, *}$, Michael R. Stark ${ }^{1,2}$ and Marianne Bronner-Fraser ${ }^{1}$ \\ ${ }^{1}$ Division of Biology and Beckman Institute, California Institute of Technology, Pasadena, CA, 91125, USA \\ 2Department of Developmental and Cell Biology, University of California, Irvine, Irvine, CA 92717, USA \\ *Author for correspondence (e-mail: cmarcell@caltech.edu)
}

\section{SUMMARY}

Shortly after their formation, somites of vertebrate embryos differentiate along the dorsoventral axis into sclerotome, myotome and dermomyotome. The dermomyotome is then patterned along its mediolateral axis into medial, central and lateral compartments, which contain progenitors of epaxial muscle, dermis and hypaxial muscle, respectively. Here, we used Wnt-11 as a molecular marker for the medial compartment of dermomyotome (the 'medial lip') to demonstrate that BMP in the dorsal neural tube indirectly induces formation of the medial lip by up-regulating Wnt-1 and Wnt-3a (but not Wnt-4) expression in the neural tube.
Noggin in the dorsal somite may inhibit the direct action of BMP on this tissue. Wnt-11 induction is antagonized by Sonic Hedgehog, secreted by the notochord and the floor plate. Together, our results show that the coordinated actions of the dorsal neural tube (via BMP and Wnts), the ventral neural tube/notochord (via Shh) and the somite itself (via noggin) mediates patterning of the dorsal compartment of the somite.

Key words: somite, dermomyotome, BMP, Sonic Hedgehog, Wnt, Noggin, chick

\section{INTRODUCTION}

Somites first condense from the segmental plate mesenchyme to form epithelial balls of undifferentiated cells surrounding a cavity, the somitocoele. Within hours, the somite undergoes extensive changes along its dorsoventral and mediolateral axes (reviewed in Christ and Ordahl, 1995; Cossu et al., 1996), differentiating ventrally into the sclerotome (which will give rise to axial bones and cartilage) and dorsally into the dermomyotome (which subdivides to form dermis and skeletal muscle).

The dermomyotome is then patterned along the mediolateral axis into medial, central and lateral compartments, which contain progenitors of epaxial muscle, dermis and hypaxial muscle, respectively. Hypaxial muscle precursors differentiate only after extensive migration to the limb and body wall. Epaxial (back) muscle precursors begin differentiation earlier, with MyoD expression initiating in a few somitic cells adjacent to the neural tube as the somites first condense. At approximately the fifth most-recently formed somite, myotome formation and growth is initiated when the MyoD-positive cells apposed to the neural tube detach and come to lie under the dermomyotome. Concomitantly, precursors within the medial compartment of the dermomyotome (the 'medial lip') start involuting under the dermomyotome, initiate MyoD expression and withdraw from the cell cycle (Denetclaw et al., 1997). Through this continuous process, myotome growth is observed as a lateral expansion of MyoD expression in the somite (Cossu et al., 1996).

The medial lip of the dermomyotome is an important structure for fate determination of epaxial myotome. It represents not only the site of myotomal induction and terminal mitosis but also the site of the involution of myotomal precursors (Denetclaw et al., 1997). In early somite development, the medial lip is the sole source of muscle precursors; later, mitotically competent muscle precursors, expressing the fibroblast growth factor receptor FREK, invade the myotomal compartment of the somite (Marcelle et al., 1995).

Some of the molecular and tissue interactions that segregate the somite into sclerotome and myotome have been elucidated. The notochord and floor plate (via Sonic hedgehog) can induce expression of Pax-1 in prospective sclerotomal cells (Fan and Tessier-Lavigne, 1994; Johnson et al., 1994). In vitro and in vivo studies have indicated that the notochord (via Sonic hedgehog) and the dorsal neural tube (via Wnt-1) can induce muscle differentiation, assayed by MyoD expression and myosin heavy chain expression (Munsterberg et al., 1995; Stern et al., 1995; Pownall et al., 1996).

The nature of the molecular interactions responsible for the induction of the dermomyotome has been elusive. At the tissue level, ectoderm (via a contact-mediated factor) and dorsal neural tube (via a soluble factor) can induce formation and/or maintenance of the dermomyotome (Gallera, 1966; Fan and Tessier-Lavigne, 1994; Spence et al., 1996). It was recently demonstrated that BMP-4 in the lateral plate mesoderm regulates patterning of the dermomyotome, resulting in the formation of the lateral compartment of the dermomyotome (Pourquié et al., 1996). However, no information is available regarding the molecular interactions that regulate the induction 
of the medial compartment of the dermomyotome, the 'medial lip'.

In this study, we used microsurgical manipulations and ectopic expression of various signaling molecules in the avian embryo to determine the tissue and molecular interactions that are responsible for patterning of the dorsal compartment of the somite in vivo. We establish Wnt-11 as a specific marker of the medial lip of the dermomyotome and demonstrate that BMP signaling in the dorsal neural tube is necessary for its induction. We find that BMP activity is not direct but is mediated by Wnt-1 and Wnt-3a, also expressed in the dorsal neural tube. Interestingly, expression of noggin in the dorsomedial somite may prevent the direct action of BMP on the somite. Finally, we show that BMP/Wnt-mediated patterning of the dermomyotome can be antagonized by Sonic hedgehog, secreted by the notochord and the floor plate. Together, our results implicate the coordinated actions of the dorsal neural tube, ventral neural tube/notochord and somite itself in patterning the dorsal compartment of the somite.

\section{MATERIALS AND METHODS}

\section{Cloning of chick Wnt-11 and in situ hybridization}

We have isolated the full coding sequence of the chick Wnt-11 by RTPCR, using 2- to 3-day-old chick total RNA as template. We chose two sets of nested oligonucleotide primers within the published Wnt11 sequence (Tanda et al., 1995; GenBank accession number: D31901) to amplify the gene (inner primers: 5'CCATGGAGCCGAGCCCGCAATTTTTC; 3'GAATTCATTTGCAGACGTATCTCTC. Outer primers: 5'CTTTCTGAAAGTGGGGGACG; 3': ATCTCAGCTCAGCAGACGAG). The amplified fragment was cloned into a Gem-T vector (Promega) and sequenced. Antisense DIG-labeled RNA probes were prepared as described (Nieto et al., 1996) and used for in situ hybridization (Henrique et al., 1995). The probes used in this study were: a $1 \mathrm{~kb}$ chick Wnt-11 clone, which encompasses its entire coding sequence, isolated as described above; a $1 \mathrm{~kb}$ chick BMP-4 probe (Duprez et al., 1996); a 900 bp cDNA clone coding for a fragment of the chick Patched (Marigo et al., 1996); a 1100 bp cDNA clone coding for a fragment of the chick noggin (Connolly et al., 1997); $400 \mathrm{bp}$ cDNA clones coding for fragments of chick Wnt-1, Wnt-3a and Wnt-4 (Hollyday et al., 1995).

\section{Transfection of chick primary fibroblasts and injection of} cells in vivo

Primary fibroblasts from line 0 chicken (SPAFAS) were transfected with retroviral constructs. One week later, the culture was totally infected as judged by staining with the polyclonal antibody recognizing the viral protein p27 (SPAFAS). Infected fibroblasts or cell lines were isolated with trypsin and allowed to recover for one hour at room temperature in $20 \%$ foetal bovine serum-containing medium. They were centrifuged and resuspended in a solution containing 10\% sucrose, 10\% CM-DiI (Molecular Probes) and 10\% ethanol. After a 15 minutes incubation at $37^{\circ} \mathrm{C}$, the cells were resuspended in a large volume of Ringer buffer and pelleted. In each experiment, injection of parental cells served as negative controls. These were: primary chick fibroblasts when retrovirally infected cells were injected, $\mathrm{CHO}$ DHFR $^{-}$cells for noggin-producing cells and MV7 Rat-2 cells for Wnt-1-expressing cells.

\section{RESULTS}

\section{Wnt-11 is a molecular marker of the dorsal somite}

Here, we establish Wnt-11 (Tanda et al., 1995; Christiansen et al., 1995) as a molecular marker for the medial lip of the dermomyotome and use its expression to follow signals critical for dermomyotome patterning. Wnt-11 expression is first observed in the medial portion of the most rostral somites at stage 14 (described in Tanda et al., 1995; Fig. 1A-C). Its expression expands caudally with embryonic age, but does not extend caudal to the fifth most recently formed somite. Thus, Wnt-11 expression is initiated after the dermomyotome has formed; its field of expression defines a molecularly distinct compartment in the dermomyotome, the medial lip. By comparing similarly staged embryos hybridized with $M y o D$ or Wnt-11, we observed that initiation of Wnt-11 expression follows that of $M y o D$ and corresponds to the start of myotome formation and growth as indicated by the lateral expansion of $M y o D$ expression (Fig. 1C). At later embryonic stages (> stage $20)$, Wnt-11 is observed in the medial lip of the dermomyotome, as well as in the dermis and the mesenchyme between the neural tube and the medial lip (Tanda et al., 1995; not shown).

\section{The dorsal neural tube and the notochord regulate induction of Wnt-11 in the medial lip}

The restriction of Wnt-11 expression at the medial edge of the dermomyotome could be the result of positive signaling from
Fig. 1. (A,B) Wnt-11 is expressed in the dorsal lip of the dermomyotome. (A) Stage 20 embryo hybridized with an RNA probe for Wnt-11. Strong Wnt-11 expression is observed in the mediodorsal portion of the somite (A). (B) In cross section at the level of the dotted line, Wnt11 expression is restricted to the medial-most portion of the dermomyotome, the 'medial lip'. NT, neural tube; N, notochord. (C) Wnt-11 expression correlates with formation of the myotome. Embryos were hybridized with $M y o D$ $(n=24)$ or Wnt-11 (n=23). The initiation of Wnt11 (red dots) and MyoD (blue open circles) were compared by plotting the embryonic age in
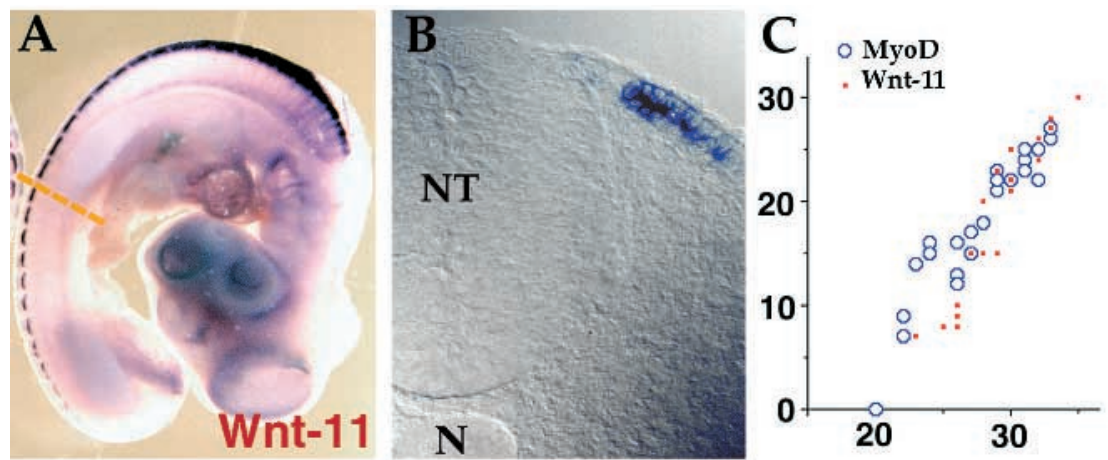
Different values for a specific embryonic somites do not display Wnt-11 expression or lateral expansion of the MyoD domain (although all somites are MyoD-positive at their medial edge). At the 30-somite stage, the 22 rostral-most somites are Wnt-11-positive and display lateral expansion of $M y o D$. In general, we observed that the expression of Wnt-11 overlaps with $M y o D$ expansion, indicating its correlation with myotome formation and growth. 
midline structures (neural tube and/or notochord), or from negative signals emanating from lateral stuctures (lateral somite, intermediate mesoderm or lateral plate mesoderm). Separating the segmental plate from the lateral plate mesoderm or sectioning the segmental plate along its midline did not change the Wnt-11 expression pattern one day later (not shown), indicating that Wnt-11 expression is not regulated by negative signals emanating from the lateral mesoderm. Rather, Wnt-11 might be regulated by factor(s) present in the neural tube. Ablation of the dorsal neural tube adjacent to the segmental plate or epithelial somites prior to the onset of Wnt11 expression (Fig. 2A) resulted in the absence of Wnt-11 one day following the operation (Fig. 2A-C). In the reciprocal experiment, the paraxial mesoderm was separated from most of the neural tube and notochord, leaving only a small piece of the ipsilateral dorsal neural tube attached (Fig. 2D); one day after surgery, this manipulation resulted not only in the induction but also the enhancement of Wnt-11 expression on the ipsilateral compared with the contralateral side (Fig. 2D-F). These results indicate that the dorsal neural tube is necessary for induction of Wnt-11 expression. In addition, the enhancement of Wnt-11 expression observed in the latter experiment suggests that the ventral neural tube and/or notochord might contain a negative regulator of Wnt-11 induction, since these tissues are separated from the somite by the experimental manipulation. Grafting a notochord between the neural tube and the paraxial mesoderm eliminated Wnt-11 expression in the medial lip of the somite at the level of the graft (Fig. 2G,H), confirming that the notochord can negatively regulate Wnt-11.

\section{BMP and Wnt in the neural tube regulate dermomyotome patterning}

The dorsal neural tube expresses numerous signaling molecules implicated in patterning of the neural tube (Hogan, 1996; Hollyday et al., 1995) and the somites (Stern et al., 1995; Munsterberg et al., 1995), including members of the TGF $\beta$ family (BMP-4 and BMP-7) and of the Wnt family (Wnt-1, Wnt-3a and Wnt4). BMP-4 expression in the dorsal neural tube precedes that of Wnt-11 in the medial lip of the somite (Reissmann et al., 1996; Fig. 3A,B). To test the possibility that BMP-4 signaling is required for Wnt-11 expression, we first inhibited its activity. The secreted factor noggin was recently shown to physically bind to and inactivate BMP-4 and, to a lesser extent, BMP-7 (Zimmerman et al., 1996; Holley et al., 1996). Therefore, we injected noggin-expressing cells into the paraxial mesoderm of developing embryos. The noggin-expressing cells were injected at axial levels prior to the observation of Wnt-11 expression in the somites. One day after injection, Wnt-11 expression was totally abolished in the vicinity of the injected cells, whereas control cells had no effect. This result indicates that a BMP signal might be required for induction of Wnt-11 in the medial lip of the dermomyotome.

To test whether BMP expression is sufficient to induce Wnt11 expression, we injected BMP-4-producing cells into the neural tube. This exogenous source of BMP-4 caused enhanced Wnt-11 expression in the medial lip of the dermomyotome immediately adjacent to BMP-expressing cells (Fig. 3D-E). In contrast, control cells had no effect. However, over-expression of BMP-4 in the neural tube did not extend the normal field of Wnt-11 expression (compare Figs $1 \mathrm{~B}$ and $3 \mathrm{E}$ ). Moreover, injection of BMP-4-producing cells into the lateral portion of the somites also failed to induce Wnt-11 expression in other
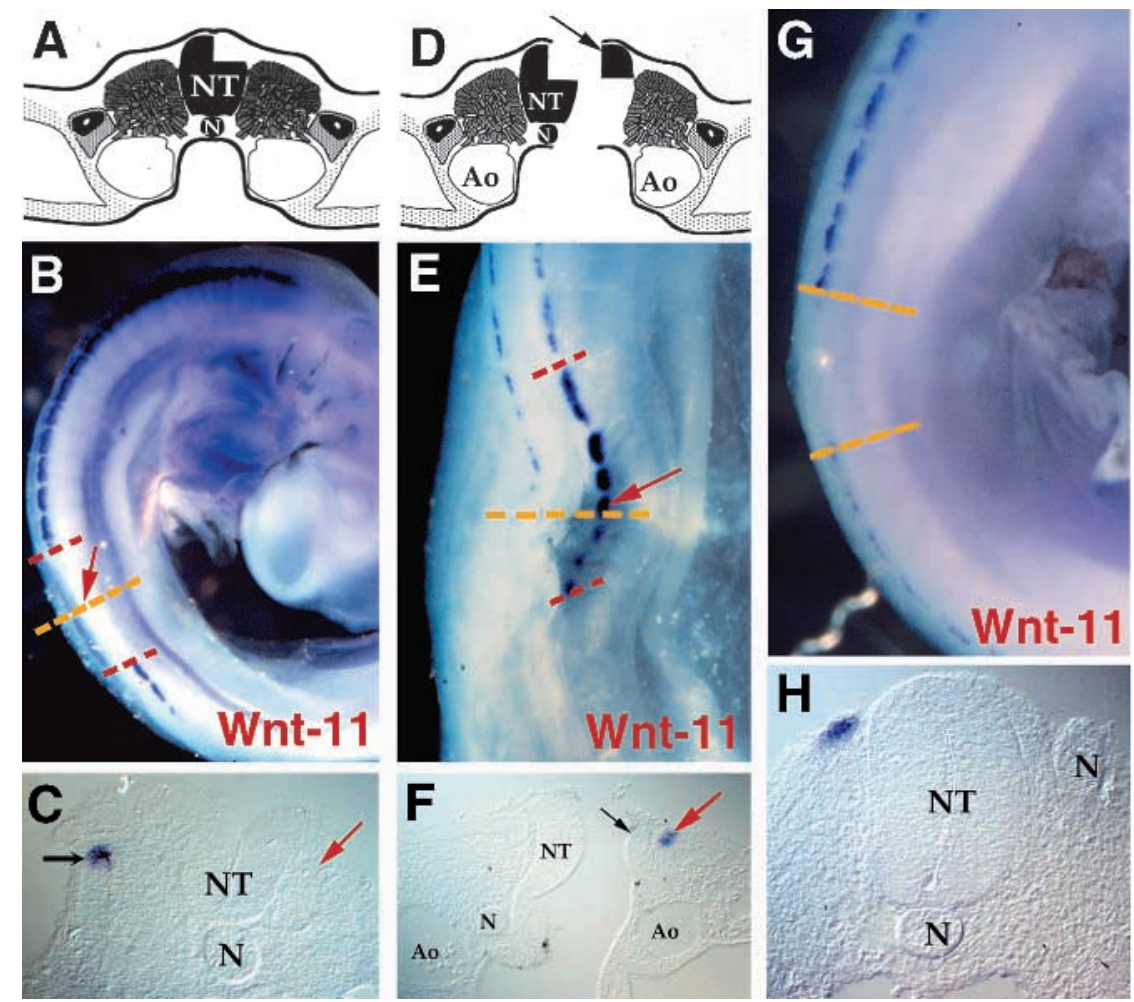

Fig. 2. (A-C) The dorsal neural tube is necessary for Wnt-11 expression in the dorsal lip. Dorsal neural tube of stage 11-13 embryos was ablated $(n=5)$ as described in BronnerFraser (1996) at the level of the segmental plate or the newly formed somites, i.e. before Wnt-11 expression is initiated (A). One day later (B), embryos lacked Wnt-11 expression on the operated side of the embryo (red arrow). (C) A transverse section at the level of the yellow dotted line in B confirmed the absence of Wnt-11 expression (red arrow), while the control side (black arrow) is normal. (D-F) The dorsal neural tube is sufficient for Wnt-11 expression. The segmental plate or the newly formed somites of stage 11-13 embryos were surgically separated from all but the dorsal neural tube (D). The paraxial mesoderm in the region of surgery was allowed to develop under the patterning influence of the dorsal neural tube (D,F, black arrow). One day later $(n=7)$, Wnt-11 expression was enhanced on the operated side of the embryo (E,F, red arrow), when compared to the control side. Red dotted lines indicate the extent of the surgery. $(\mathrm{G}, \mathrm{H})$ The notochord inhibits Wnt-11 expression in the dorsal lip. Notochords isolated from the early somitic level of stage 12-13 embryos were grafted in stage-matched hosts at the level of the tip of the segmental plate, into a slit made between the neural tube and the paraxial mesoderm. One day later $(\mathrm{G}), W n t-11$ expression was absent $(n=12)$ in the region of the grafted notochord (delineated by the dotted lines). A transverse section $(\mathrm{H})$ in this region confirmed the absence of Wnt-11 in the vicinity of the grafted notochord. N, notochord; NT, neural tube; Ao, aorta. 

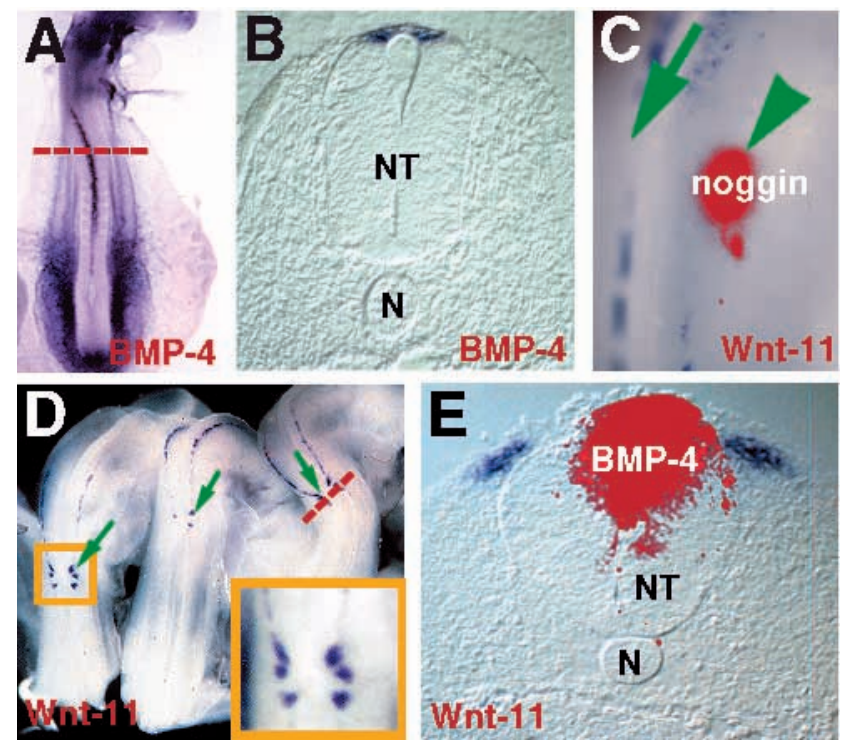

Fig. 3. (A,B) BMP-4 is expressed in the dorsal neural tube. In situ hybridization of a stage 13 (20-somite) embryo with a chick BMP-4 RNA probe (A) showed that this molecule is highly expressed in the neural tube adjacent to the level of the rostral segmental plate onward to the most rostral somites. A transverse section (B) at the level of the dotted line showed that BMP-4 is expressed in the dorsal neural tube. At this developmental stage, Wnt-11 is not yet expressed in the dorsal somite. (C) Noggin inhibits induction of Wnt-11 in the dorsal lip. DiI-labeled CHO cells transfected with a Xenopus noggin construct were injected at multiple levels in the paraxial mesoderm (at the level of the fifth newly formed somite, the rostral end of the segmental plate and Hensen's Node, i.e. before Wnt-11 expression is initiated) of stage 13-14 chick embryos $(n=10)$. One day later, Wnt11 expression was absent around the injected cells (indicated by the red patch). While Wnt-11 expression was absent on the ipsilateral, injected side (arrow), its expression was reduced on the contralateral side as well (arrowhead), indicating that noggin secreted by the injected cells can act at a distance. (D,E) BMP-4 over-expression enhances Wnt-11 expression in the dorsal lip. BMP-4-expressing chick primary fibroblasts, produced by infection with a mouse BMP4 retroviral construct, were injected $(n=4)$ into the lumen of the neural tube of stage 10-11 embryos roughly at the level of the fifth most recently formed somite. One day later, Wnt-11 expression was enhanced in two or three somites directly adjacent to the injected cells (indicated by arrows in D), when compared to somites rostral and caudal to the site of injection. $\mathrm{E}$ is a composite of a bright-field image of a transverse section at the level of the dotted line (in D) and a fluorescent image of the same section showing the brightly DiIlabeled BMP-4-expressing cells. NT, neural tube; N, nototchord.

locations than the dorsomedial somite (not shown). Together, these results indicate that BMP signaling is necessary but not sufficient for ectopic induction of Wnt-11. It is possible that Wnt-11 expression results from the combination of BMP and other signals in the dorsal neural tube. Alternatively, BMP may regulate the expression of another molecule(s) in the dorsal neural tube, which in turn directly activates Wnt-11 expression.

To distinguish between these possibilities, we tested the ability of members of the Wnt family to induce Wnt-11 expression in the dorsal somite alone or in combination with BMP. Similar to BMP-4, expression of Wnt-1, Wnt-3a and $W n t-4$ in the dorsal neural tube precedes that of Wnt-11 in the
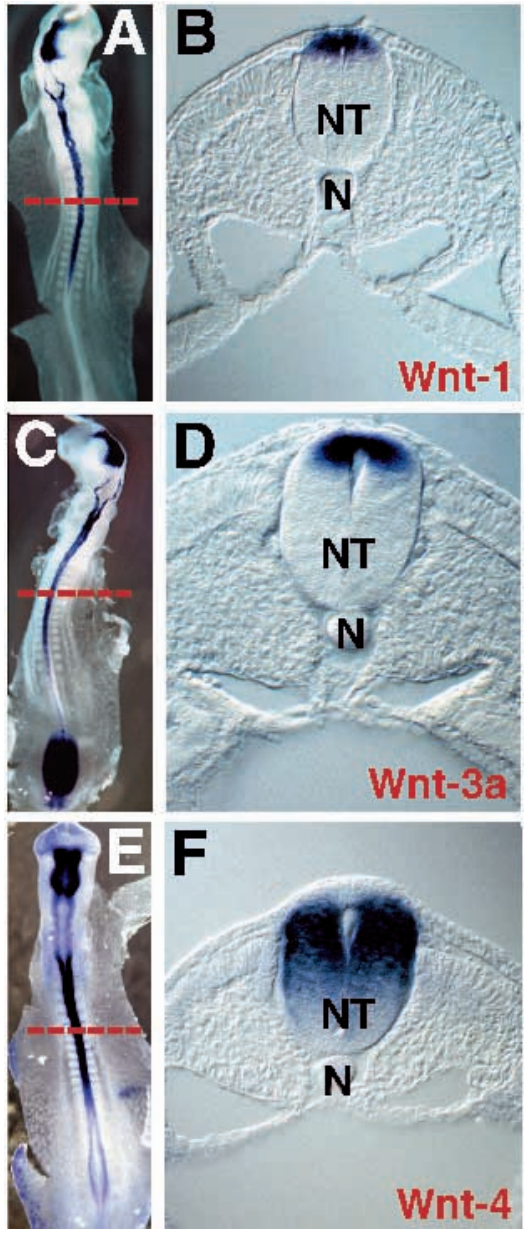

Fig. 4. Wnt-1, Wnt-3a and Wnt-4 are expressed in the dorsal neural tube. In situ hybridization of stage 12 (15-17 somites) embryos with chick Wnt-1 (A,B), Wnt-3a (C,D) and Wnt-4 (E,F) probes showing that these molecules are expressed in the dorsal neural tube roughly at the level of the first newly formed somite, i.e. before Wnt-11 starts being expressed in the medial lip of the dermomyotome. NT, neural tube; N, notochord.

medial lip of the somite (Hollyday et al., 1995; Fig. 4A-F). We found that Wnt-1-expressing cells injected into the paraxial mesoderm of developing embryos induced ectopic expression of Wnt-11 throughout the entire dermomyotome, while control cells did not affect its expression (Fig. 5 A, B).

To test whether Wnt-1 expression is regulated by BMP, we injected BMP-producing cells in the lumen of the neural tube of developing embryos. One day later, the domain of Wnt-l expression in the neural tube was expanded ventrally around the BMP-4-expressing cells (Fig. 5C,E) when compared to its normal expression away from the injected cells (Fig. 5D). Conversely, injection into the paraxial mesoderm of cells expressing the BMP-inhibiting molecule noggin reduced or totally abolished Wnt-1 expression in the neural tube (Fig. 5F). Similarly, Wnt-3a expression was down-regulated by noggin (Fig. 5G). Together, these experiments indicate that BMP signaling regulates $W n t-1$ and $W n t-3 a$ expression in the dorsal neural tube, which in turn induce Wnt-11 in the dermomyotome. Surprisingly, BMP signaling differentially regulates members 
Fig. 5. (A,B) Wnt-1 induces ectopic expression of Wnt-11. Rat-2 cells infected with a mouse Wnt-1 construct were injected in the paraxial mesoderm of developing embryos. One day later, we observed lateral expansion of $W n t-11$ expression (A). A transverse section at the level of the dotted line (B) shows ectopic expression of Wnt-11 throughout the entire dermomyotome. (C-E) In the neural tube, BMP-4 induces expansion of the Wnt- 1 expression domain BMP-4-expressing cells were injected into the lumen of the neural tube of stage 10-11 embryos roughly at the level of the fifth most recently formed somite. One day later, the domain of Wnt-1 expression was expanded ventrally around the BMP4-producing cells (C). When compared to the normal Wnt- 1 expression, which was restricted to the roof plate of the neural tube (D), Wnt-1 expression around the injected cells was observed throughout the dorsal third of the neural tube (E). (F-H) BMP regulates Wnt-1 and Wnt-3a, but not Wnt-4 in the neural tube. Cells expressing the BMP-inhibiting

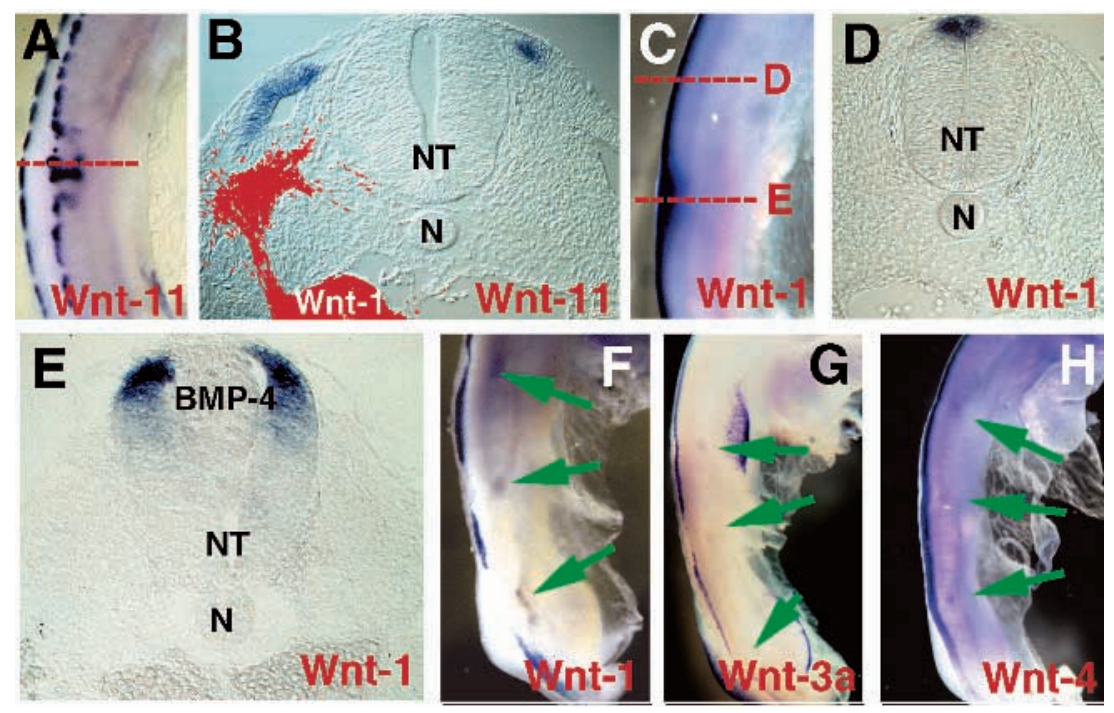
factor noggin were injected in the paraxial mesoderm of stage 12-14 (15- to 20-somite) embryos at the level of the fifth newly formed somite, the rostral end of the segmental plate and Hensen's node. One day later, Wnt-1 (F) and Wnt-3a $(\mathrm{G})$ expression was reduced or totally abolished around the injected cells (indicated by an arrow). In contrast, Wnt-4 (H) expression was unaffected. NT, neural tube; N, notochord.

of the Wnt family in the neural tube; in contrast to Wnt-1 and Wnt-3a, Wnt-4 expression was unaffected by noggin (Fig. 5H).

\section{Noggin in the medial somite may inhibit direct effects of BMP on myotome formation}

While our results show that BMP in the neural tube is important for patterning of the medial dermomyotome, previous findings demonstrate that ectopic expression of BMP in the somites can inhibit MyoD expression (Pourquié et al., 1996; our observation). Therefore, proper somite patterning might require the absence of a BMP signal within the dorsal somite. This raises the possibility that an inhibitor of BMP may be present in the somite. One potential BMP inhibitor is noggin.

To test whether endogenous noggin has the proper tissue distribution to act as an inherent inhibitor of BMP function within the dorsal somite, chick embryos having 15-40 somites were hybridized with a chick noggin cDNA probe. In young embryos (15-25 somites), noggin was expressed in a striking pattern in the dorsomedial portion of the developing somites, in close proximity to the BMP-4-producing neural tube (Fig. 6A-C; compare Figs $3 \mathrm{~B}$ and $6 \mathrm{C}$ ). In the newly formed somites, noggin was expressed laterally (Fig. 6D), becoming progressively more medial in more rostral somites. With increasing embryonic age, noggin expression eventually disappears from the medial (but not from the lateral) somite in a caudal-rostral fashion: in embryos with 35-40 somites, noggin is no longer expressed medially (not shown).

Noggin in the dorsomedial somite could affect not only the action of BMP on the somites, but also on the neural tube. To test this, we separated the neural tube of 15- to 20-somite-stage embryos from the somites, which express noggin at this developmental stage; after 8 hours, no difference in the BMPregulated Wnt- 1 expression was observed in the neural tube in the region of the surgery compared with rostral or caudal regions (not shown), indicating that noggin might act locally in the somite.

\section{Sonic hedgehog inhibits Wnt-11 in the dermomyotome}

Sonic hedgehog (Shh), which is expressed by the notochord
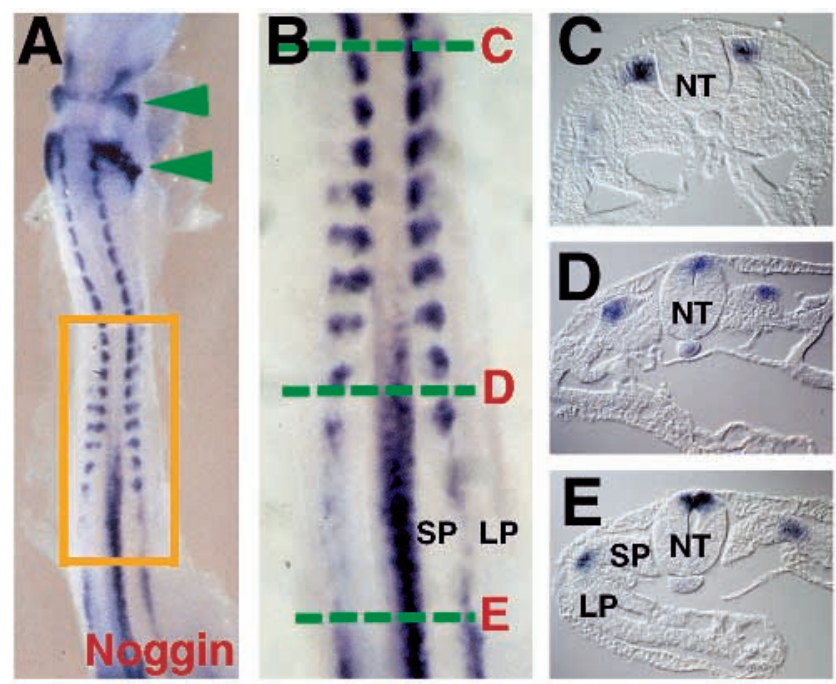

Fig. 6. Noggin is expressed in the medial somite of developing embryos. (A-E) A 17-somite embryo hybridized with a chick noggin probe. (B) Enlargement of the area bordered in A. In all, but the newly formed somites, noggin was expressed at the dorsomedial border of the somite (as shown in C). At the level of the 5-7 newly formed somites, noggin expression gradually shifted to the lateral portion of the somite (D). At this level, noggin was also present in the dorsal neural tube, and faintly in the notochord. At the level of the segmental plate $(E)$, robust noggin expression was observed in the dorsal neural tube and in the intermediate mesoderm which separates the segmental plate and the lateral plate mesoderm. In addition to somitic expression, noggin transcripts were observed in the dorsal neural tube and the notochord at the level of the segmental plate, as well as in the neural crest exiting from rhombomeres 4 and 6 (arrowheads in A) at the hindbrain level. NT, neural tube; SP, segmental plate; LP, lateral plate. 


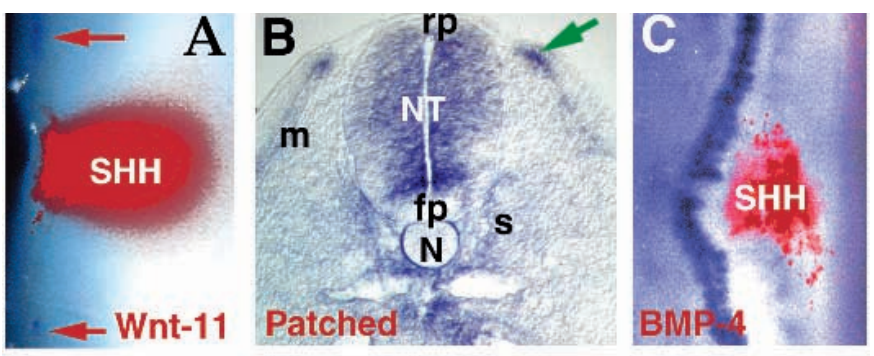

Fig. 7. (A) Sonic hedgehog inhibits Wnt-11 induction in the dorsal lip. Primary fibroblasts infected with a Shh-carrying retrovirus were injected in the paraxial mesoderm of stage 10-12 embryos $(n=8)$. Two days later, Wnt-11 expression was absent in somites surrounding the injected cells (red patch). Red arrows indicate normal Wnt-11 expression rostral and caudal to the injected cells. (B) Expression of the sonic hedgehog receptor Patched in the dorsal somite. A 34-somite embryo was hybridized with a probe coding for the Sonic hedgehog receptor Patched. Patched expression was observed in the medial sclerotome (s) which surrounds the notochord $(\mathrm{N})$, in the myotome $(\mathrm{m})$ and in the medial lip of the dermomyotome (shown by an arrow). In addition, robust Patched expression was observed throughout the neural tube (NT), with the exception of the roof plate (rp) and the floor plate (fp). (C) Sonic hedgehog does not alter BMP-4 expression in the neural tube. Sonic hedgehog-producing cells were injected $(n=5)$ in the paraxial mesoderm of stage 10-12 embryos. One day later, BMP-4 expression in the neural tube was normal around the injected cells (in red). In the same experimental series, the Shh-producing cells induced overexpression of Pax-1, as previously described (Johnson et al., 1994; Fan and Tessier-Lavigne, 1994; not shown).

and floor plate, can mimic the notochord's inhibition of Wnt11 expression. Injection of DiI-labeled Shh-producing fibroblasts into the paraxial mesoderm totally abolished Wnt-11 expression in the vicinity of the fibroblasts two days later (Fig. 7A). In contrast, control cells had no effect. It has been shown in explant studies that endogenous Shh can function at a considerable distance from its source (Fan and Tessier-Lavigne, 1994). Therefore, the notochord and the ventral neural tube (via a Shh-mediated signal) in vivo may actively repress Wnt-
11 expression except at the most dorsal dermomyotome. Although we cannot unequivocally distinguish between direct Shh effects on the somite versus indirect effects mediated by the neural tube (as shown by Pourquié et al., 1993), we observed that the Shh receptor Patched is expressed in the medial lip of the dermomyotome (Fig. 7B), raising the possibility of a direct receptor/ligand interaction in the medial lip. Consistent with a direct effect of Shh on dermomyotome patterning, the injection of Shh-producing cells did not alter the pattern of BMP-4 expression in the neural tube (Fig. 7C).

\section{Absence of Wnt-11 expression correlates with abnormal myotome formation}

Manipulations that result in loss of Wnt-11 expression, such as ablation of the dorsal neural tube or injection of Shh-producing cells into the somite, result in the disorganization of the myotome. For example, unilateral ablations of the dorsal neural tube in the segmental plate region of developing embryos (as shown in Fig. 2A) result in an apparent failure of involution of dermomyotomal cells through the medial lip of the dermomyotome (Fig. 8A), from which Wnt-11 is absent. MyoDpositive cells that differentiate at the level of the first somites failed to involute, but remain adjacent to the ablated neural tube; in contrast, on the unoperated side, they both involuted and expanded laterally under the dermomyotome.

Injection of Shh-expressing cells into the paraxial mesoderm also results in the absence of Wnt-11 expression (Fig. 7A). After this manipulation, MyoD-positive cells were totally disorganized rather than arranged longitudinally as in a normal myotome (Fig. 8B and inset). In addition to a considerable expansion of the $M y o D$-expressing cell population (as previously described by Johnson et al., 1994; Fig. 8B,C), nearly the entire dermomyotome, which normally does not express $M y o D$, was $M y o D$-positive (Fig. 8B), suggesting that the cells initiate myogenesis despite their failure to involute. A morphologically recognizable dermomyotome containing a few MyoD-positive cells is observed peripheral to the injection site (not shown). These results suggest that Shh over-expression and the associated loss of Wnt-11 expression result in the
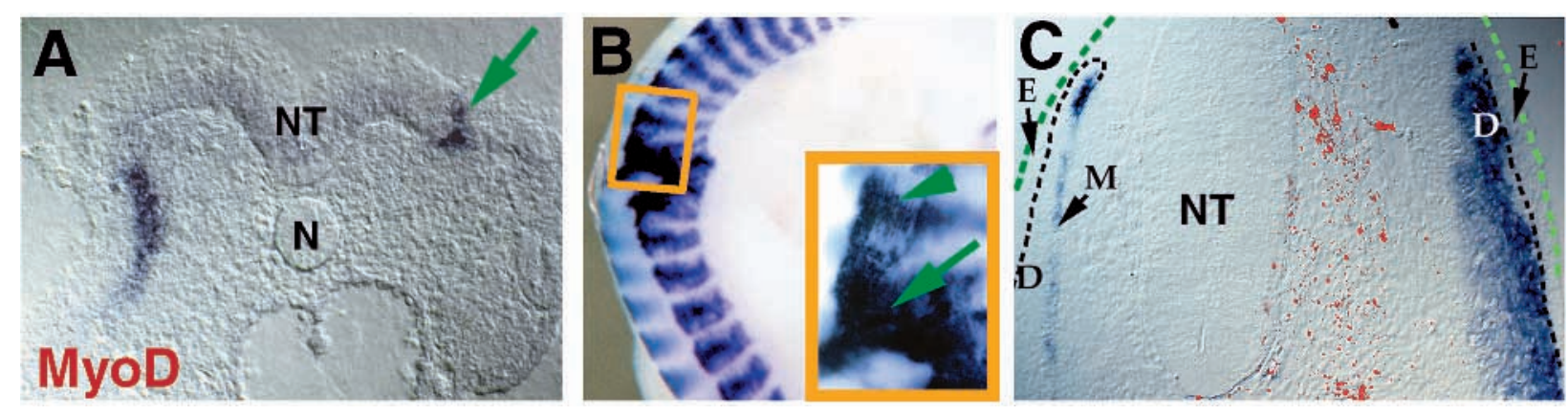

Fig. 8. MyoD expression after ablation of the dorsal neural tube. Ablation of the dorsal neural tube leads to a failure of involution of $M y o D$ positive cells; note that the MyoD-positive myotome failed to involute and expand laterally on the injected (arrow) side compared with the noninjected (left) side. MyoD-positive cells differentiated as a compact mass which remained adjacent to the ablated neural tube. (B,C) Sonic hedgehog-mediated Wnt-11 inhibition alters myotome formation. Sonic hedgehog-producing cells were injected in the paraxial mesoderm of stage 10-12 embryos $(n=4)$. Two days later, embryos were processed by in situ hybridization for MyoD. (B) In whole mount, the distinct expansion of the $M y o D$-positive cell population was obvious in the vicinity of the injected cells. In addition, myocytes were disorganized (arrow) rather than arranged longitudinally as they were rostral and caudal to the injected cells (arrowhead). (C) Comparing the control (left) and injected (right) myotome, $M y o D$-positive cell population adjacent to the injected cells (in red) was expanded when compared to normal myotome and myotomal differentiation was observed throughout the dermomyotome ( $\mathrm{D}$, delineated by a black dotted line). The border of the ectoderm is indicated by a dotted green line. NT, neural tube. 


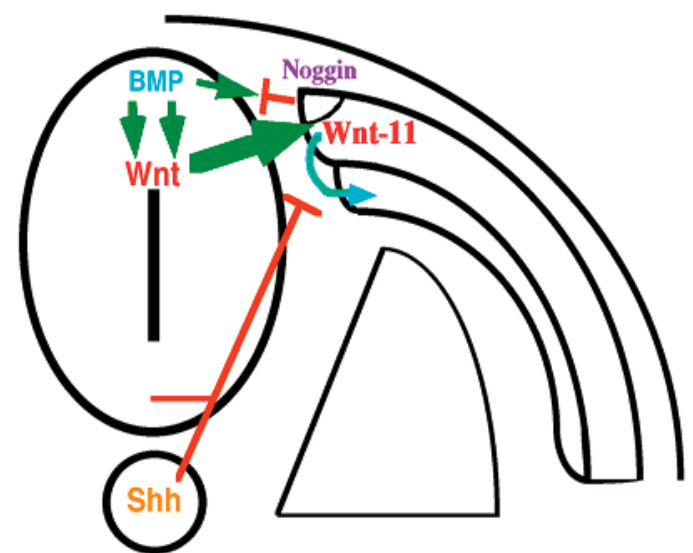

Fig. 9. A model of BMP-mediated patterning of the dermomyotome. BMP regulates Wnt (Wnt-1 and Wnt $3 a$ ) expression in the dorsal neural tube, which in turn induces Wnt-11 in the medial lip. Wnt-11 expression allows muscle progenitors present in the dermomyotome to involute into the myotome (blue arrow). Noggin in the medial somite prevents BMP from directly acting on the paraxial mesoderm. Sonic hedgehog, acting as an antagonist of BMP-mediated Wnt-11 induction, represses Wnt-11 expression in its immediate vicinity, keeping Wnt-11 expression dorsal.

absence of involution of myotomal precursor cells and their subsequent disorganized differentiation into myocytes within the dermomyotome.

\section{DISCUSSION}

Within hours of their formation, somites undergo extensive changes along their dorsoventral axis, differentiating ventrally into the sclerotome and dorsally into the dermomyotome. The sclerotome, as assayed by the expression of Pax-1, forms and/or expands under the influence of Sonic Hedgehog (secreted by the floor plate and the notochord). In contrast, the nature of the molecular interactions responsible for the initial induction of the dermomyotome have yet to be determined. It was shown that ectoderm can induce formation of the dermomyotome (Gallera, 1966), which in turn is maintained by factor(s) from the dorsal neural tube (Fan and Tessier-Lavigne, 1994; Spence et al., 1996).

Soon after its formation, the dermomyotome is patterned along its mediolateral axis into medial, central and lateral compartments. Lineage studies have recently demonstrated that the medial compartment (or 'medial lip') contains the progenitors of the epaxial muscle (Denetclaw et al., 1997). Our observation that Wnt-11 is a molecular marker of the medial lip of the dermomyotome enabled us to analyze the tissue and molecular interactions responsible for its formation. We demonstrated that the dorsal neural tube (via BMP and Wnt) mediates the formation of the medial lip of the dermomyotome. Concomitantly, the lateral plate mesoderm induces the differentiation of the lateral dermomyotome, resulting in expression of Sim-1 (Pourquié et al., 1996) and Met (Bladt et al., 1995), and enhanced levels of Pax-3 (Goulding et al., 1994; Williams and Ordahl, 1994) before their migration into the limb or trunk abdominal muscles. Intriguingly, the formation of a lateral compartment in the dermomyotome is also regulated by BMP-
4, expressed by lateral plate mesoderm (Pourquié et al., 1996). Therefore, depending on the cellular and molecular context in which it acts, BMP-4 plays a dual role: produced by the dorsal neural tube, it indirectly induces multipotent dermomyotomal cells (Ordahl and Le Douarin, 1992) to differentiate into Wnt11 -expressing medial lip cells and may play a role in myotome organization; in the lateral plate mesoderm, BMP-4 induces differentiation of dermomyotomal cells into Sim-1/Metexpressing cells and represses their myogenic differentiation.

We present the first evidence that BMP differentially regulates the expression of various Wnt molecules. The Wnt family can be subdivided into two distinct subclasses, based on their ability to induce double axis in Xenopus or to inhibit activin-induced convergence-extension movements (Du et al., 1995). Wnt-1 and Wnt-3a belong to one subclass and are regulated by BMP in our study, while Wnt-4 (and Wnt-11) belong to the second subclass, which does not seem to be affected by BMP. The regulation of Wnt-1/3a by BMP-4 is consistent with previous findings demonstrating that BMP can regulate Wnt during frog mesoderm induction (Graff et al., 1994). An appealing hypothesis is that BMP molecules can differentially induce members of the two subclasses and suggests that this could be a novel regulatory mechanism implicated in multiple developmental paradigms in which various members of the TGF $\beta$ and Wnt families are co-expressed.

Noggin, which was shown to antagonize BMP activity during frog gastrulation, may play a role in regulating BMP patterning of the somite. The pattern of expression of noggin in the dorsomedial somite explains some apparently paradoxical activities of BMP. On the one hand, we show that BMP is required for Wnt- 1 and Wnt-3a expression in the dorsal neural tube, which in turn can promote muscle differentiation (Stern et al., 1995; Munsterberg et al., 1995). On the other hand, ectopic expression of BMP-4 in the paraxial mesoderm results in the inhibition of myotome formation (Pourquié et al., 1996; our observation). Therefore, it is important for proper myotomal development that the BMP protein be withheld from the paraxial mesoderm. Multiple mechanisms might sequester BMP within the neural tube, including a selective permeability of the basement membrane that surrounds the neural tube and/or a strong affinity of BMP to extracellular matrix molecules surrounding the neural tube cells. We show that, in addition to these potential barriers, the presence of the BMPinhibiting molecule noggin in the dorsal somite is consistent with it acting as a potential repressor of BMP molecules that diffuse away from the neural tube. At present, we cannot discriminate whether the neural tube activity is mediated by BMP4, BMP-7 or both, since noggin inhibits both molecules. Noggin is also expressed in the lateral portion of the most caudal somites and in the intermediate mesoderm at the level of the segmental plate. In these locations, noggin is directly apposed to strong BMP-4-expressing domains, and is likely to regulate and/or to prevent BMP-4 activity on the paraxial mesoderm.

The expression of Wnt-11 expression in the medial lip of the dermomyotome correlates with proper organization of the myotome. Because Wnt-11 expression occurs at the site of ingression of myotomal precursors, it is tempting to speculate that the absence of Wnt-11 expression in the medial lip of the somite after either neural tube ablation or injection of Shh-cells may be responsible for the myotomal disorganization and the 
failure of the muscle progenitors to ingress into the myotome. Specific knock-out of Wnt-11 expression will be needed to test this possibility directly and differentiate between the primary and secondary effects of our manipulations. During the morphogenetic movements of gastrulation, Wnt-11 in the blastopore lip of the frog blastopore (Ku and Melton, 1993) has been shown to be important for the conversion-extension movements (Du et al., 1995). The movements of the ectoderm through the blastopore lip are somewhat analogous to the ingression occurring during myotome formation; thus, it is possible that Wnt-11 plays similar role during these two dynamic processes. Wnt-11 is not required for initial induction of the myotome, since expression of $M y o D$, the earliest marker of muscle differentiation in chick, precedes its expression. Moreover, we show that formation of the myotome and of the medial lip of the dermomyotome are separable inductive events, since $M y o D$ induction occurs in the absence of Wnt-11 expression.

While the patterning activities of Shh, Wnts and TGF $\beta$ family members have been well documented in developmental processes as diverse as Xenopus gastrulation to patterning of the Drosophila leg imaginal disc, it is interesting to note that the molecular interactions that regulate somite patterning are not necessarily parallel to those observed in other organisms or even in other tissues within the same organism. For example, establishment of the anterior-posterior axis in the leg imaginal disc is mediated by interactions between Hedgehog, Wingless and Dpp. During this process, Hedgehog activates both Wingless and Dpp (Penton and Hoffmann, 1996). In contrast, we show that Sonic Hedgehog does not affect the transcription of the $D p p$-like molecule BMP-4, while it inhibits the Wingless-like molecule Wnt-11. In the leg imaginal disc, Dpp inhibits Wingless (Jiang and Struhl, 1996). In contrast, we show that BMP activates Wnt-1 and Wnt-3a, which in turn induce Wnt-11. Furthermore, during chick limb patterning, it was established that Wnt-7a activates Sonic Hedgehog (Yang and Niswander, 1995). Conversely, we show that Sonic hedgehog is able to inhibit Wnt-11.

We propose the following model for patterning of the medial dermomyotome (Fig. 9). The expression of Wnt-11 in the medial lip is the result of a balance of inducing interactions from the dorsal neural tube and inhibitory interactions from the ventral neural tube/notochord. BMP in the dorsal neural tube induces expression of Wnt-1 and Wnt-3a (but not Wnt-4), which in turn mediate(s) expression of Wnt-11 in the medial lip of the dermomyotome. Wnt-11 expression may allow muscle progenitors in the medial lip to involute into the myotome. Noggin within the somites restricts the action of BMPs. Finally, Shh, secreted by the floor plate and notochord, represses Wnt-11 expression in its immediate vicinity, restricting its expression to the dorsal component of the somite. The balance of these interactions leads to proper patterning of the medial dermomyotome and subsequent differentiation into the myotome.

We thank Drs Charles Ordahl and Scott Fraser for critical reading of the manuscript and Johnny Choi and Sung Hee Kil for their help during this research. We are grateful to the following for their generous gifts of reagents used in this study: Dr Paul Brickell for the chick BMP-4 probe used for in situ hybridization and the mouse BMP-4 cDNA cloned in the RCAS(A) retroviral vector, Dr Richard
Harland for the noggin-secreting CHO cells, Dr Cliff Tabin for the chick Sonic hedgehog cDNA cloned in the RCAS(A) retroviral vector and for the chick Patched probe, Dr Bruce Patterson for the chick $M y o D$ probe, Dr Anthony Brown for the Wnt-1-expressing cell line and Dr Jonathan Cooke for the chick noggin probe. This work was supported by grants from the Muscular Dystrophy Foundation to C. M. and M. B. F.

\section{REFERENCES}

Bladt F., Riethmacher D., Isenmann S., Aguzzi A. and Birchmeier C. (1995). Essential role for the c-met receptor in the migration of myogenic precursor cells into the limb bud. Nature 376, 768-771.

Bronner-Fraser, M. (1996). Manipulations of neural crest or their migratory pathways. In Methods in Cell Biology 51, 61-79. (ed. M. Bronner-Fraser). New York: Academic Press.

Christ B. and Ordahl C. (1995). Early stages of chick somite development. Anatomy and Embryology 191, 381-396.

Christiansen J., Dennis C., Wicking C., Monkley S., Wilkinson D. and Wainwright B. (1995). Murine Wnt-11 and Wnt-12 have temporally and spatially restricted expression patterns during embryonic development. Mech. Dev. 51, 341-350.

Connolly, D., Patel, K. and Cooke, J. (1997). Chick noggin is expressed in the organizer and neural plate during axial development, but offers no evidence of involvement in primary axis formation. Int. J. Dev. Biol. 41, 389-396.

Cossu G., Tajbakhsh S. and Buckingham M. (1996). How is myogenesis initiated in the embryo? Trends in Genetics 12,218-223.

Denetclaw, W., Christ, B. and Ordahl, C. (1997). Location and growth of epaxial myotome precursor cells. Development 124, 1601-1610..

Du, S., Purcell, S., Christian, J., McGrew, L. and Moon, R. (1995). Identification of distinct classes and functional domains of Wnts through expression of wild-type and chimeric proteins in Xenopus embryos. Mol. Cell. Biol. 15, 2625-2634.

Duprez D., Bella E., Richardson M., Archer C., Wolpert, L., Brickell, P. and Francis-West, P. (1996). Overexpression of BMP-2 and BMP-4 alters the size and shape of developing skeletal elements in the chick limb. Mech. Dev. 57,145-157.

Fan C. M. and Tessier-Lavigne M. (1994). Patterning of mammalian somites by surface ectoderm and notochord evidence for sclerotome induction by a hedgehog homolog. Cell 79, 1175-1186.

Gallera, J. (1966). Mise en évidence du role de l'ectoblaste dans la différenciation des somites chez l'oiseau. Revue Suisse de Zoologie, 73, 492503

Goulding M., Lumsden A. and Paquette A. (1994). Regulation of Pax-3 expression in the dermomyotome and its role in muscle development. Development 120, 957-971

Graff J., Thies R., Song J., Celeste A. and Melton D. (1994). Studies with a Xenopus BMP receptor suggest that ventral mesoderm-inducing signals override dorsal signals in vivo. Cell 79, 169-179.

Henrique D., Adam J., Myat A., Chitnis A., Lewis J. and Ish-Horowicz D. (1995). Expression of a Delta homologue in prospective neurons in the chick. Nature 375,787-790.

Hogan B. (1996). Bone morphogenetic proteins: multifunctional regulators of vertebrate development. Genes Dev. 10,1580-1594.

Holley, S., Neul, J., Attisano, L., Wrana, J., Sasai, Y., O’Connor, M., De Robertis, E. and Ferguson, E. (1996). The Xenopus dorsalizing factor Noggin ventralizes Drosophila embryos by preventing DPP from activating its receptor. Cell 86, 607-617.

Hollyday M., McMahon J., McMahon A. (1995). Wnt expression patterns in chick-embryo nervous-system. Mech. Dev. 52, 9-25.

Jiang, J. and Struhl, G. (1996), Complementarity and mutually exclusive activities of Decapentaplegic and Wingless organize axial patterning durin Drosophila leg development. Cell 86, 401-409

Johnson R., Laufer E., Riddle R. and Tabin C. (1994). Ectopic expression of Sonic Hedgehog alters dorsal-ventral patterning of somites. Cell 79, 1165 1173.

Ku, M. and Melton, D. (1993). X-Wnt-11: a maternally expressed Xenopus Wnt gene. Development 119, 1161-1173.

Marcelle C., Wolf J. and Bronner-Fraser M. (1995). The in vivo expression of the FGF receptor FREK messenger-RNA in avian myoblasts suggests a role in muscle growth and differentiation. Dev. Biol. 172, 100-114.

Marigo V., Scott M., Johnson R., Goodrich L. and Tabin, C. (1996). 
Conservation in hedgehog signaling: induction of a chicken patched homolog by Sonic hedgehog in the developing limb. Development 122, 1225 33.

Munsterberg A., Kitajewski J., Bumcrot D., McMahon A. and Lassar A. (1995). Combinatorial signaling by Sonic hedgehog and Wnt family members induces myogenic bHLH gene-expression in the somite. Genes Dev. 9, 2911-2922.

Nieto, A., Patel, K. and Wilkinson, D. (1996). In situ hybridization analysis of chick embryos in whole mount and tissue sections. In Methods in Cell Biology 51, 220-235. (ed. M. Bronner-Fraser). New York: Academic Press.

Ordahl, C. and Le Douarin, N. (1992). Two myogenic lineages within the developing somite. Development 114, 339-353.

Penton, A. and Hoffmann, F. (1996). Decapentaplegic restricts the domain of wingless during drosophila limb patterning. Nature 382, 162-165

Pourquié O., Coltey M., Teillet M.-A., Ordahl C. and Le Douarin N. (1993). Control of dorsoventral patterning of somitic derivatives by notochord and floor plate. Proc. Natn. Acad. Sci. USA 90, 5242-5246.

Pourquié O., Fan C., Coltey M., Hirsinger E., Watanabe Y., Bréant, C., Francis-West, P., Brickell, P., Tessier-Lavigne, M. and Le Douarin, N. (1996). Lateral and axial signals involved in avian somite patterning: a role for BMP4. Cell 84, 461-471.

Pownall M., Strunk K. and Emerson C. (1996). Notochord signals control the transcriptional cascade of myogenic bHLH genes in somites of quail embryos Development 122, 1475-1488.
Reissmann E., Ernsberger U., Francis-West P., Rueger D., Brickell P. and Rohrer, H. (1996). Involvement of bone morphogenetic protein-4 and bone morphogenetic protein-7 in the differentiation of the adrenergic phenotype in developing sympathetic neurons. Development 122, 2079-2088.

Spence, M., Yip, J. and Erickson, C. (1996). The dorsal neural tube organizes the dermomyotome and induces axial myocytes in the avian embryo. Development 122, 231-241.

Stern H., Brown A. and Hauschka S. (1995). Myogenesis in paraxial mesoderm: preferential induction by dorsal neural-tube and by cells expressing Wnt-1. Development 121, 3675-3686.

Tanda N., Ohuchi H., Yoshioka H., Noji S. and Nohno T. (1995). A chicken Wnt gene, Wnt-11, is involved in dermal development. Bioch. Bioph. R. 211, 123-129.

Williams B. and Ordahl C. (1994). Pax-3 expression in segmental mesoderm marks early stages in myogenic cell specification. Development 120, 785796.

Yang, Y. and Niswander, L. (1995). Interaction between the signaling molecules $w n t 7 a$ and Shh during vertebrate limb development - dorsal signals regulate anteroposterior patterning. Cell 80, 939-947

Zimmerman L., Dejesusescobar J. and Harland R. (1996). The Spemann organizer signal Noggin binds and inactivates bone morphogenetic protein-4. Cell 86, 599-606. 